\title{
Heurs et malheurs des langues finno-ougriennes de Russie
}

ФИННО-УГОРСКИЙ МИР: ЯЗЫКОВЫЕ СТРАДАНИЯ И РАДОСТИ

The Finno-ugric world: Good and Bad Linguistic Fortunes

РОССИЯЛЭН ФИННО-УГОР ДУННЕЕЗ: КЫЛ КУРАДЗОНЪЁС НО ШУМПОТОНЪЁС

\section{Aleksej Arzamazov}

Traducteur : Dominique Samson Normand de Chambourg

\section{(2) OpenEdition}

\section{Journals}

Édition électronique

URL : https://journals.openedition.org/efo/1624

DOI : $10.4000 /$ efo. 1624

ISSN : 2275-1947

\section{Éditeur}

INALCO

\section{Édition imprimée}

Date de publication : 1 décembre 2013

ISBN : 978-2-343-04446-0

ISSN : 0071-2051

\section{Référence électronique}

Aleksej Arzamazov, « Heurs et malheurs des langues finno-ougriennes de Russie », Études finnoougriennes [En ligne], 45 | 2013, mis en ligne le 03 février 2015, consulté le 08 juillet 2021. URL : http:// journals.openedition.org/efo/1624; DOI : https://doi.org/10.4000/efo.1624

Ce document a été généré automatiquement le 8 juillet 2021.

\section{(c) (†) \&}

Études finno-ougriennes est mis à disposition selon les termes de la Licence Creative Commons Attribution - Pas d'Utilisation Commerciale 4.0 International. 


\title{
Heurs et malheurs des langues finno-ougriennes de Russie
}

\author{
ФИННО-УГОРСКИЙ МИР: ЯЗЫКОВЫЕ СТРАДАНИЯ И РАДОСТИ \\ The Finno-ugric world: Good and Bad Linguistic Fortunes \\ РОССИЯЛЭН ФИННО-УГОР ДУННЕЕЗ: КЫЛ КУРАДЗОНЪЁС НО ШУМПОТОНЪЁС
}

Aleksej Arzamazov

Traduction : Dominique Samson Normand de Chambourg

\section{I.}

1 Vers la fin du xxe siècle, on dénombrait quelque 6000 langues à travers le monde, avec parmi elles une bonne moitié de micro-langues dont le destin au cours du siècle à venir s'annonce tragique. Le livre des langues en danger existe déjà, et de nouveaux noms s'y ajoutent chaque année. La disparition des cultures, dont la langue est un aspect fondamental, s'explique le plus souvent par toutes sortes de raisons: globalisation, unification ethnoculturelle, processus d'assimilation, etc. Les sociétés dont il s'agit n'ont alors presque plus de force de résistance à opposer : les hommes sont occupés à de tout autres jeux. La politique du diktat économique menée par l'écrasante majorité des États ne prend guère en compte les «intérêts » ethnoculturels et linguistiques. Elle a même des effets pervers, remplaçant les structures internes stables par d'autres, externes et fluctuantes. Il n'est pas étonnant que la rhétorique superficielle du consensus, pivot et slogan premier du discours politique général, ne s'inscrive pas dans la réalité des choses. Sur la carte du monde, les foyers de conflits armés et culturels continuent furieusement de s'étendre. Des peuples entiers deviennent otages de leur hétérodoxie, s'ils font valoir leur droit de ne pas vivre selon les lois de la globalisation, de la prétendue « démocratie ». La langue devient l'objet de manipulations, elle obtient de facto le statut d'arme. Les catégories de connaissance et de méconnaissance ne relèvent plus de l'opposition entre le soi et l'autre, mais deviennent un appel ouvert à agir... 
2 Il ne faut pas oublier que la langue est un système ("synergétique ») des plus complexes, un système mobile. Les vecteurs de ce mouvement sont divers : progressifs, régressifs, synchroniques, diachroniques. Les facteurs de pression, eux, peuvent à ce niveau ne pas être dominants : parfois, la langue, avec la spécificité de son système explicatif, s'éteint, disparaît d'" elle-même ». De ce point de vue, les « grandes » langues sont, à première vue, hors de danger. Quel risque pourraient bien courir l'anglais et le russe? En dépit de la différence des trajectoires de ces deux langues (la première "gagne» toujours plus de locuteurs sur les autres, la seconde perd nombre des siens), l'une comme l'autre font pourtant face à un problème aigu: la sauvegarde et le maintien de leur propre «qualité » culturelle. Le problème est accru par l'amplitude géographique, par l'absence d'une ligne politique unie et d'un même scénario social d'optimisation de la qualité de la langue.

3 Parallèlement au processus de disparition des langues et d'appauvrissement de leur thésaurus culturel, émerge au sein même des langues un processus de dialectalisation (ainsi, la variante cantonaise du chinois se distingue résolument du baïhua) et une différenciation croissante des formes orale et écrite. Au début du $\mathrm{xxl}^{\mathrm{e}}$ siècle, le développement des langues s'accompagne de simplifications grammaticales partielles et d'une fermeture au lexique international. Plus dynamiques qu'avant, bilinguisme, trilinguisme, voire multilinguisme, se propagent.

4 Il y a beaucoup à dire sur l'ethnolinguistique dans le monde contemporain. Avant de passer à l'exposé de quelques observations dans le cadre d'un essai relativement libre, regardons une hypothétique mappemonde linguistique.

5 Vers 2050, selon les données de The English Company UK, le quinté de tête serait le suivant, en nombre de locuteurs: chinois (putonghua, dialectes hou, yue, min, sian, hakka, gan), 1384 millions de locuteurs ; hindi et ourdou, autrefois considérés comme une langue unique par les chercheurs (l'actuelle distinction découle de raisons politiques, mais aussi de systèmes graphiques différents et d'influences lexicales diverses, sanskrit d'un côté, arabe et persan de l'autre), 556 millions; anglais, 508 millions; espagnol, 486 millions; arabe, 482 millions. Pour les autres langues majeures, leur évolution serait la suivante : russe, 275 millions ; bengali, 215 millions ; portugais, 194 millions ; français, 129 millions ; allemand, 128 millions ; japonais, 126 millions ; coréen, 75 millions ; javanais, 75 millions ; telugu, 75 millions, etc. ${ }^{1}$

6 Toutes ces langues se trouvent en relations antinomiques avec des langues voisines, particulièrement en cas de territoire commun. Le chinois "empiète» sur les langues tibétaines; l'aïnou s'est déjà dilué dans le japonais; l'anglais assimile ou "sape » la portée des langues les plus diverses, qu'elles soient celtiques, indiennes, ou africaines ; l'espagnol s'oppose également aux langues indiennes (en premier lieu, au quechua) et complique l'existence du basque. Autrement dit, l'accroissement de certaines cultures linguistiques en fait reculer d'autres. Or ces langues sur le recul constituent une majorité passive, condamnée à une triste fin, délétère pour l'œcuménisme humain.

7 Dans la Russie contemporaine, comme naguère dans l'espace de l'Union soviétique, on observe la coexistence du grand-russe et de nombreuses langues «régionales ». Les données du recensement de $2002^{2}$, porteuses du pathos de "l'union linguistique nationale », montrent ouvertement « les traumatismes culturels et linguistiques » de la société russienne ${ }^{3}$ : à de rares exceptions près, les «autres" langues deviennent au mieux peu à peu étrangères à leur propre sphère ethnoculturelle, au pire elles tombent 
dans la désuétude jusqu'à n'être plus qu'un mot du dictionnaire antédiluvien, rapidement oublié.

8 Le destin des langues finno-ougriennes de Russie, dont il sera question ici, est intermédiaire, il s'articule entre les deux "conditions » évoquées. Le nombre de locuteurs des plus grandes d'entre elles (le mordve-erza et le mokcha, l'oudmourte, les langues komies, zyriène et permiak) diminue sensiblement. Les langues les moins pratiquées (le khanty, le mansi, le vepse, le nenetse, le same, l'ingrien et le vote) sont sur le point de disparaître. Il est déjà vraisemblablement trop tard pour les sauver : le déclin de la mosaïque linguistique semble irréversible.

II.

Comment les sciences humaines peuvent-elles étudier les fondements linguistiques et culturels du modèle ouralien (finno-ougrien) ? Il faut non seulement prêter attention aux processus « locaux » dans les microsystèmes linguistiques producteurs de culture (par exemple l'étude de l'étymologie dans un texte de littérature orale), mais aussi accomplir des "voyages sémiotiques" dans la sphère des autres realia macrolinguistiques. La recherche des niveaux linguistiques locaux (par exemple le descriptif dialectologique, la différenciation phonétique des parlers) doivent être combinées avec des arguments de caractère philosophique très abstraits.

Malgré l'apparente latence de ses "archives culturelles », la langue est, nous l'avons déjà noté, une matière vivante, mobile, changeante. La réaction d'une langue vivante, le regard conventionnel de l'expérience collective passée sur le présent et le futur, constituent des thèmes qui intéressent la linguistique et la psychologie contemporaines et qu'elles soulèvent régulièrement. Si nous tenons compte de la constance et de l'invariabilité de la réaction de la langue aux facteurs sociaux, nous pouvons espérer être en mesure de reconstruire les contours, et parfois les tournants décisifs, de l'influence sociétale sur la langue, et vice-versa. On ne peut ignorer dans ce contexte l'importance du créateur individuel (le poète, par exemple), enclin à expérimenter, à «flirter » avec la langue, et par là même apte à influer sur la situation linguistique et culturelle. Eu égard à ce qui précède, la question de l'invariabilité ontologique ou, à l'inverse, de la mobilité du modèle est justifiée.

11 En observant le modèle linguistico-culturel qui sous-tend la vision oudmourte du monde par rapport aux langues apparentées et voisines, on trouvera sans doute nombre de traits communs, on mettra en évidence des typologies parallèles dans le développement linguistique, au niveau des structures superficielles et profondes. Pourtant, certains détails essentiels, des «différentiels", préservent encore l'oudmourte et sa matrice archaïque des " heurts et dérives » linguistiques touchant l'humanité. Dans ce qui suit, nous donnons des exemples d'affranchissement culturel ou d'érosion interne - évidente ou non-, en oudmourte et dans les autres langues finno-ougriennes, hier et aujourd'hui.

12 De notre point de vue, en comparaison avec les langues des autres communautés finnoougriennes de Russie, la langue oudmourte et son corpus lexical actuel sont un exemple de «neutralité linguistico-culturelle». Cette neutralité est perceptible dans le processus "d'observation participative", à la recherche de catégories conceptuelles qui n'existent pas dans l'immémorial univers linguistique oudmourte, mais dont la présence est causée par la pression informative du russe et, par son intermédiaire, par 
la globalisation contemporaine. Il ne s'agit pas seulement d'une construction linguistique artificielle et du renforcement des mots nouveaux dans la conscience collective.

13 L'oudmourte est une langue de symbioses relatives. Préservé d'une totale tatarisation (l'influence, elle, est bien réelle!) et, semble-t-il, en adéquation avec un fonds général russe, il n'a pas perdu ses modèles immanents de communication, «préservant » la carte oudmourte du monde de l'interférence radicale de ses habiles voisins, grands ou petits. En outre, la modernisation passe encore par un fonds lexical et morphologique immémorial amenant à réduire les emprunts étrangers - qui, en général, ne s'inscrivent guère dans la vie intérieure de la langue, de ses mots et de ses expressions.

Du point de vue de son fonctionnement socioculturel, l'oudmourte occupe également une position intermédiaire entre les langues finno-ougriennes européennes, utilisées aux niveaux les plus divers, et les langues sur le point de disparaître. Il ne faut certes pas s'attendre à un renforcement de la position de l'oudmourte dans son milieu ethnoculturel d'origine. Malheureusement, la langue autochtone cesse d'être dominante dans la conscience de la plupart de ses locuteurs - jeunes ou personnes d'âge moyen. L'affaiblissement de la culture du discours se lit dans les œuvres de la littérature oudmourte contemporaine, qui véhiculent de nombreux clichés grammaticaux non oudmourtes. Il faut dire que c'est dans la première moitié du $\mathrm{xx}^{\mathrm{e}}$ siècle que la création en oudmourte était autosuffisante. On peut noter aujourd'hui une crise de la formation des mots. Les sources se tarissent ; les suffixes manquent. Les possibilités de formation de formes substantivées (-on/ën, -os, -ès, -èt/et, -ni, -či, -lyk) ne permettent pas un perfectionnement lexical suffisant, ni de compenser la dynamique de la production terminologique, d'autant que certains des termes nouveaux sont en contradiction avec les lois phonétiques de l'oudmourte et brisent le rythme de sa "chaîne sonore ». Élargir le corpus lexical oudmourte, déjà inadéquat dans différents domaines (sciences humaines, médecine), requiert l'apport de la philologie et une mobilisation sociale considérable. La linguistique contemporaine n'est sans doute que partiellement apte à résoudre les problèmes linguistiques cruciaux (mais il y a des exemples de réactivation réussie de langues). Dans le cas de l'oudmourte, plus encore que le travail réfléchi des linguistes, il faut trouver des méthodes non standard et élaborer des projets transethniques. Par exemple, on pourrait créer des archives lexicales permiennes (komi-oudmourtes), dont les matériaux reposeraient essentiellement sur les nécessités d'une politique conceptuelle de production de vocabulaire.

Les autres langues finno-ougriennes de Russie « définissent » également à leur manière le degré de dépendance du substrat, les mesures qualitatives et quantitatives de son autonomie, de sa fermeture ou de son "orientabilité». Le carélien, avec sa riche histoire écrite et littéraire, tend à « l'adéquation contemporaine » au finnois et perd en partie son originalité stylistique traditionnelle. Le vepse, l'ingrien, le vote, longtemps confinés dans un blocus socioculturel absolu, meurent ou intègrent petit à petit l'espace linguistique finno-estonien contemporain (capacité lexicographique, uniformité syntaxique). L'erza, dans son aire dialectale d'usage courant, tend à se russifier à grande vitesse et acquiert les horizons lexicaux et syntaxiques du russe. Ce processus se voit par exemple dans la littérature orale collectée dans l'oblast de Nižnij Novgorod, avec un poème au lexique mi-erza, mi-russe. 

s'abîment dans leurs immémoriales racines totémiques et mythologiques. Parfois, il est vrai, elles jaillissent de livres richement édités, à la couverture flamboyante. Le nenetse, éloigné des « macrocivilisations » linguistiques, conserve sa matrice grâce aux «océans» des toundras. Le mari, en particulier les formes des plaines et de l'Est, a prêté l'oreille aux suggestions lexicales du tatar et du bachkir et se trouve par là même sous leur aile protectrice. Comme le montrent les recensements de 1989 et 2002, seul le mari, parmi les langues finno-ougriennes de Russie, présente une évolution positive.

Historiquement dans l'orbite du russe-pomor septentrional, le komi-zyriène semble avoir modifié ses vecteurs de développement du côté d'un clavier lexicodialectologique propre. Néanmoins, on peut difficilement douter de la dualité linguistique de la vision traditionnelle komi-zyriène du monde. Son bilinguisme ethnoculturel apparaît par exemple dans la coexistence du komi et du russe dans la littérature orale.

À l'évidence, le champ linguistique et culturel a un lien ontogénétique avec une série de processus psychologiques; la connaissance de la langue est condamnée à un rapprochement avec la psychologie. La disparition, le changement radical des «rythmes internes» des langues finno-ougriennes contemporaines de Russie, sont directement liés à la "température " du temps, à la mobilité et à la destruction de configurations autrefois immuables, au changement considérable de la compétence communicationnelle de l'être humain.

\section{III.}

Il existe aujourd'hui un projet linguistique international qui suscite un vif écho dans le monde finno-ougrien, et qui a pour noble but de rapprocher les langues : la création d'une langue finno-ougrienne commune. La communauté finno-ougrienne a un avis très partagé sur la question. Les uns y voient le résultat de la souffrance des "petites " langues, les autres voient dans cette langue, le « boudinos », un manifeste de résistance politique; d'autres encore saluent l'apparition d'une langue finno-ougrienne commune et lui prédisent un grand avenir.

Le projet intéresse nombre de pays, dont les pays finno-ougriens, la Finlande, la Hongrie et l'Estonie, mais aussi l'Allemagne, la France, les Pays-Bas, l'Italie, l'Espagne, la Communauté des États indépendants, les États-Unis, le Canada, le Mexique, l'Argentine, l'Arabie Saoudite, la Chine, le Japon ainsi que les républiques de Polynésie. Le boudinos, cette langue commune des Finno-ougriens, a déjà permis d'écrire des œuvres littéraires; une série de recensions et d'articles lui a été consacrée. Grâce au compositeur estonien Mart Simmer, la langue a acquis une dimension symphonique, avec la présentation, le 4 juin 2009, de la suite Mina upin jos, en l'église Saint-Jean de Tartu. À l'heure actuelle, des musiciens oudmourtes préparent un projet à grande échelle, dans le cadre duquel le boudinos sera une langue chantée. En divers endroits $\mathrm{du}$ monde, on commence à se saluer avec les mots de Paro lona et Paro volide. L'université oudmourte d'État a prévu des cours de boudinos ; la liste d'attente est déjà longue. L'engouement est bien réel: la langue est objet d'intérêt de la part des créateurs, mais aussi d'un sentiment de responsabilité - on veut et on peut continuer à jouer avec elle, à partager observations et impressions... 
Depuis deux siècles, l'humanité joue à produire des langues artificielles et, visiblement, ne s'en lasse pas. Cela ne surprend guère. Le goût des langues, déjà, est une réalité ; ensuite, jouer avec une "nouvelle" langue peut remporter un vif succès dans une perspective communicationnelle. Une langue artificielle peut parfois approcher un but clair, mais ambitieux : faciliter l'entregent. Il y a toutefois un grand nombre de " mais ", objectifs et subjectifs, dont il faut tenir compte, qui conditionnent la complexe relation sociale à de tels projets.

Je ferai remarquer que l'auteur de ces lignes ${ }^{4}$ n'appartient pas au groupe des utopistes malheureux; au contraire, il s'efforce de regarder lucidement le phénomène des langues artificielles, en chercheur. La réalisation de ce projet lui a permis d'emblée de regarder le monde linguistique dans une autre perspective, d'étudier l'histoire de la linguistique et la carte des peuples finno-ougriens, de croiser le passé et de ressentir en même temps la complexité du présent. Quelqu'un pourrait demander : « Qu'en est-il de l'avenir?» Préjuger du futur est intéressant, mais vain. Cela relève, on le sait, du domaine des pronostics. J'ignore sincèrement ce qu'il adviendra du boudinos. Son existence dépend de nombreux facteurs. Il est important de comprendre que l'actualité d'une langue finno-ougrienne commune réside dans l'originalité d'un œcuménisme finno-ougrien partagé sur les continents les plus divers. Notre parenté se reflète précisément dans le miroir de la langue, alors que les dimensions sociale et culturelle n'y trouvent guère de points communs.

23 Quelle langue doit parler un Finno-ougrien du $\mathrm{xxI}^{\mathrm{e}}$ siècle vivant en Russie? Tout d'abord, la sienne. La sauvegarde de sa propre langue est la tâche essentielle à l'ordre du jour finno-ougrien. Le boudinos apparaît comme un simple prétexte à une convivialité « légère » et joyeuse, si celle-ci est encore possible. Certains ont dit qu'il était trop complexe pour une langue artificielle, mais ce n'est qu'une illusion de complexité. Certes, il est un peu plus ardu que d'autres langues artificielles, mais sa nature est autre. Il est, si l'on veut, à la fois sanscrit et esperanto ; il combine en lui des formes lexicales archaïques, établissant ainsi un pont concret entre ethnies éloignées, mais plutôt qu'une reconstruction, le boudinos est le résultat d'une modélisation. Il est simple, logique, sa grammaire est réduite au minimum de règles nécessaires. Début 2010, deux livres ont été publiés, qui familiarisent le lecteur avec cette langue finnoougrienne commune. On peut espérer que ce n'est qu'un début. Le lexique est encore en chantier, la grammaire est à polir. L'écriture se perfectionne. Son aspect étrange, pas tout à fait finno-ougrien, est lié à la réalité de nos claviers : le clavier anglais est sur n'importe quel ordinateur. Par son emprunt de la variante anglaise de l'alphabet latin, le boudinos convient parfaitement à l'utilisation active de l'ordinateur.

\section{IV.}

24 À l'évidence, dans la vie des langues finno-ougriennes de Russie comme dans l'existence des gens ordinaires, coexistent heurs et malheurs. Il reste à regretter que les joies se fassent de plus en plus rares, et les malheurs beaucoup plus présents... 


\section{NOTES}

1. Voir www.ethnologue.com

2. Un nouveau recensement a eu lieu en 2010 (NdT).

3. Dans l'article, ce terme fera écho à Российскии, et par extension à РоссиАнин (resp. adjectif et substantif), c'est-à-dire relevant de l'espace russe, indifféremment de l'ethnie (NdT).

4. Auteur lui-même du « boudinos » (NdR).

\section{RÉSUMÉS}

Dans cet essai, l'auteur exprime quelques idées sur les processus externes et internes en cours dans les langues finno-ougriennes de Russie en ce début de $\mathrm{xx}^{\mathrm{e}}$ siècle. Il se penche sur certains phénomènes réguliers qui caractérisent la situation des langues minoritaires et permettent d'apprécier la mosaïque du monde finno-ougrien par le prisme de l'intuition philologique. Chercheur et poète, Aleksej Arzamazov met ici en évidence, sans se borner à une stricte interprétation scientifique, quelques satisfactions et quelques souffrances que suscite la situation actuelle de l'oudmourte et discute des possibilités et des perspectives des projets de langues artificielles.

В ЭССЕ ОТРАЖЕНЫ НЕКОТОРЫЕ МЫСЛИ О ВНЕШНИХ И ВНУТРЕННИХ ПРОЦЕССАХ, ИМЕЮЩИХ МЕСТО В ФИННО-УГОРСКИХ ЯЗЫКАХ РОССИИ В НАЧАЛЕ ХХІ СТОЛЕТИЯ. АВТОР ОСТАНАВЛИВАЕТСЯ НА ОТДЕЛЬНЫХ НЕОЧЕВИДНЫХ ЗАКОНОМЕРНОСТЯХ И СОСТОЯНИЯХ МИНОРИТАРНЫХ ЯЗЫКОВ, ПОЗВОЛЯЮЩИХ ПОСМОТРЕТЬ НА МОЗАИКУ ФИННО-УГОРСКОГО МИРА СКВОЗЬ ПРИЗМУ ФИЛОЛОГИЧЕСКОЙ ИНТУИЦИИ. ОСТАВЛЯЯ В СТОРОНЕ СТРОГО-НАУЧНЫЕ МЕТОДЫ ИНТЕРПРЕТАЦИИ, ФИЛОЛОГ И ПОЭТ АЛЕКСЕЙ АРЗАМАЗОВ ОЧЕРЧИВАЕТ КРУГ РАДОСТЕЙ И СТРАДАНИЙ УДМУРТСКОГО ЯЗЫКА И РАССУЖДАЕТ О ВОЗМОЖНОСТЯХ И ПЕРСПЕКТИВАХ ИСКУССТВЕННЫХ ЯЗЫКОВЫХ ПРОЕКТОВ.

This essay reflects the author's thoughts about the external and internal processes that are taking place in the Finno-Ugric languages in Russia at the beginning of the 21st century. The author concentrates on some non- visible regularities and peculiarities of minority language, which allow looking at the Finno-Ugric world's mosaic from the point of view of philological intuition. Aleksej Arzamazov is both poet and scholar. Here, he leaves apart rigid scientific interpretation methods and emphasises some rejoicing and saddening features in the Udmurt experience; he reflects as well on the possibilities and perspectives of artificial language projects. 
INDEX

Index chronologique : XXIe siècle (début), XXIe siècle

Keywords : endangered languages, lexical creation, Dialects, artificial language, Budinos, Argentina, Canada, China, Commonwealth of Independent States, Estonia, Finland, France, Germany, Holland, Hungary, Italy, Japan, Mexico, Polynesia, Russia, Saudi Arabia, Spain, Tartu, USA, Siimer Mart (1967-)

Mots-clés : langues en danger, création lexicale, dialectes, langue artificielle, boudinos Index géographique : Allemagne, Arabie Saoudite, Argentine, Canada, Chine, Espagne, Estonie, Tartu, États-Unis, Finlande, France, Hongrie, Italie, Japon, Mexique, Pays-Bas, Polynésie, Fédération de Russie

\section{AUTEURS}

ALEKSEJ ARZAMAZOV

Institut de recherche de l'Académie des sciences à Ijevsk 